МАТЕМАТИЧЕСКАЯ МОДЕЛЬ ВЯЗКОСТИ ТЯЖЕЛОЙ НЕФТИ, СОДЕРЖАЩЕЙ ПРИМЕСИ КОЛЛОИДНЫХ НАНОЧАСТИЦ ОКСИДОВ МЕТАЛЛОВ

\title{
VISCOSITY MATHEMATICAL MODEL OF HEAVY OIL CONTAINING THE METAL OXIDES COLLOID NANOPARTICLES IMPURITY
}

\section{В.И. Лесин}

Институт проблем нефти и газа Российской Академии наук, г. Москва, Российская Федерация

\section{Viktor I. Lesin}

\section{Oil and Gas Research Institute, Russian Academy of Sciences, Moscow, Russian Federation e-mail: vilesin@inbox.ru}

Аннотация. Разработка месторождений с высокой вязкостью нефти значительно осложняет ее извлечение и транспортировку. Для снижения вязкости используются различные физические методы воздействия на нефть, в том числе такие, как обработка магнитным полем и воздействие скоростью сдвига (напряжением сдвига) путем создания колебаний давления в скважинной жидкости. Магнитная обработка (MO) осуществляется путем установки устройств, оборудованных магнитами, внутри скважинного оборудования и в наземных трубопроводах. Продолжительность физических воздействий определяется эмпирическим путем на основе лабораторных и промышленных экспериментов.

В связи с вышесказанным актуальным разработка математической модели, позволяющей установить общие закономерности эволюции 
вязкости при воздействии физическими полями для повышения успешности их применения.

Целью предлагаемого исследования является создание математической модели вязкости, которая устанавливает закономерности эволюции вязкости нефти во времени при воздействии на нефть скорости сдвига и магнитного поля.

Предложена система уравнений, описывающих зависимость вязкости тяжелой нефти от времени, скорости сдвига и концентрации магнитных частиц оксидов железа. Уравнения основаны на свойствах совместных агрегатов коллоидных частиц нефти и оксидов железа, имеющих структуру физических фракталов (фрактальных агрегатов), ранее изученных методами спектроскопии рассеяния. Система уравнений содержит дифференциальное уравнение с не разделяющимися переменными, не имеющее общего аналитического решения. Справедливость дифференциального уравнения подтверждена экспериментально для трех типов частных решений, соответствующих определенным физическим ситуациям.

Abstract. Development of oil fields with high viscosity of oil significantly complicates its extraction and transportation. To reduce the viscosity, various physical methods of acting on oil are used, including such as treating with a magnetic field and the impact of shear rate (shear stress) by creating pressure oscillations in the well fluid. Magnetic processing is carried out by installing devices equipped with magnets inside the downhole equipment and in onshore pipelines. The duration of physical effects is determined empirically based on laboratory and industrial experiments.

In this way, the actual development of a mathematical model that allows to establish the general laws of the viscosity evolution when exposed to physical fields to increase their application success.

The purpose of this research work is to create a mathematical model of viscosity, which establishes the laws of the oil viscosity evolution over time when exposed to oil shear rate and magnetic field. 
A system of equations describing the dependence of the heavy oil viscosity on time, shear rate and concentration of iron oxides magnetic particles is proposed. The equations are based on the joint aggregates properties colloidal oil particles and iron oxides having the physical fractals structure (fractal aggregates) previously studied by scattering spectroscopy. The system of equations contains a differential equation with non-dividing variables that does not have a common analytical solution. The differential equation validity is confirmed experimentally for three types of particular solutions corresponding to certain physical situations.

Ключевые слова: нефть, вязкость, скорость сдвига, фрактальный агрегат, обработка магнитным полем, теория, уравнение, зависимость от времени, магнитные частицы

Key words: oil, viscosity, shear rate, fractal aggregate, magnetic treatment, theory, equation, dependence on time, magnetic particles

\section{Введение}

Разработка месторождений с высокой вязкостью нефти значительно осложняет ее извлечение и транспортировку. Для снижения вязкости используются различные физические методы воздействия на нефть, в том числе такие, как обработка магнитным полем и воздействие скоростью сдвига (напряжением сдвига) путем создания колебаний давления в скважинной жидкости. Магнитная обработка осуществляется путем установки устройств, оборудованных магнитами, внутри скважинного оборудования и в наземных трубопроводах. Продолжительность физических воздействий определяется эмпирическим путем на основе лабораторных и промышленных экспериментов.

В связи с вышесказанным актуальным разработка математической модели, позволяющей установить общие закономерности эволюции вязкости при воздействии физическими полями для повышения успешности их применения. 
Известно, что повышение вязкости нефти при изменении термобарических условия обусловлено появлением в еe составе органических коллоидных частиц, возникающих в результате выпадения в твердую фазу высокомолекулярных компонентов нефти - асфальтенов, смол и парафинов. В [1-3] была предложена модель стационарной вязкости коллоидных систем, основанная на особенности строения агрегатов коллоидных частиц. В [4-7] было установлено, что коллоидные частицы, благодаря силам межмолекулярного притяжения, образуют агрегаты, имеющие структуру физических фракталов.

Предложенная в $[1,2]$ модель основана на учете работы сил напряжения сдвига, приложенных к поверхности фрактальных агрегатов (ФА), которые приводят к перемещению ФА во вмещающей жидкости вследствие вращения в поле градиента скорости - скорости сдвига. В полученной в $[1,2]$ формуле измеряемая в данный момент вязкость нефти зависит от концентрации ФА и их физических параметров (радиуса и размерности ФА). Модель описывала стационарное значение вязкости при данной скорости сдвига, в ней не рассматривались процессы изменения вязкости во времени, сопровождающие воздействие скорости сдвига и магнитной обработки.

Целью предлагаемого исследования является создание математической модели вязкости, которая устанавливает закономерности эволюции вязкости нефти во времени при воздействии на нефть скорости сдвига и магнитного поля.

\section{Теория и эксперимент}

Согласно [1-3] вязкость $\eta$ связана со средним радиусом ФА $\mathrm{R}$ и их концентрацией $\mathrm{n}$ соотношением:

$$
\eta=\eta_{\infty}\left(1+K a^{3} n(R / a)^{\lambda}\right) .
$$


В (1) $\eta_{\infty}-$ вязкость при полностью разрушенных агрегатах, a - радиус коллоидной частицы, $\mathrm{m}, \lambda>0$, здесь и в дальнейшем $\mathrm{K}$ - постоянные, зависящие от физических параметров ФА.

Физическая модель, на основе которой получена формула (1), основана на учете потерь энергии вмещающей жидкости на процессы перемещения, деформации и разрушения ФА. Согласно [4] физическим ФА называется кластер с размерами (R/a) > 10. Модель использует ранее установленные закономерности взаимодействия вмещающей жидкости $\mathrm{c}$ такими агрегатами.

Согласно [4-6] масса ФА M $=\mathrm{m}(\mathrm{R} / \mathrm{a})^{\mathrm{D}}(1 \leq \mathrm{D} \leq 3-$ фрактальная размерность агрегата, m - масса коллоидной частицы), величина D может зависеть от расстояния от центра инерции ФА [7], т.е. в общем случае ФА обладает мультифрактальностью: $\mathrm{D}_{\mathrm{i}}=\mathrm{const}$ для $\mathrm{r}_{\mathrm{i}} \leq \mathrm{r} \leq \mathrm{r}_{\mathrm{i}+1}$. Напряжение сдвига $\boldsymbol{\sigma}$ вмещающей жидкости направлено перпендикулярно радиусвектору r, направленному из центра инерции к поверхности ФА, т.е. скалярное произведение $\boldsymbol{\sigma} \bullet \mathbf{r}=0$. Сила вязкого трения $\mathrm{F}$, действующая на поверхность фрактального агрегата, оценивается как $\mathrm{F} \sim \eta_{\infty} \mathrm{Ga}^{2}(\mathrm{R} / \mathrm{a})^{\mathrm{m}}$ [6] $\left(\mathrm{G}=\mathrm{dV} / \mathrm{dx} \quad\left(\mathrm{c}^{-1}\right)\right.$ - градиент скорости - скорость сдвига). Сила F, приложенная со стороны жидкости к поверхности ФА, совершает работу с мощностью P FGR [1-3], что и приводит к уравнению вида (1).

Под действием сил вязкого трения происходит уменьшение размеров агрегата, что выражается в зависимости радиуса от скорости сдвига $\mathrm{G}[4,6]$ :

$$
R / a \sim\left(G_{0} / G\right)^{h}
$$

где $\mathrm{G}_{0}$ - характерный параметр, зависящий от сил взаимного притяжения коллоидных частиц;

h >0 - характерный параметр, зависящий от размерности D.

С учетом (2) зависимость стационарной вязкости от скорости сдвига приобретает вид:

$$
\eta=\eta_{\infty}\left(1+K_{1} a^{3} n\left(G_{d} G\right)^{\lambda h}\right)
$$


В формуле (1) первоначально [1-3] предполагалось, что $(\mathrm{R} / \mathrm{a})^{\lambda}$ представляет постоянную величину, соответствующую стационарному значению скорости сдвига, температуры и значения концентрации ФА-n. В данной работе предложена математическая модель, в которой предполагается, что в ходе изменения $(\mathrm{R} / \mathrm{a})^{\lambda}$ во времени уравнение (1) остается справедливым. Это соответствует ситуации состояния равновесия в коллоидной системе в ходе ее перестройки.

Согласно (1) для расчета величины вязкости $\eta$ необходимо определить величину $\mathrm{R}$ в данный момент времени $\mathrm{t}$, для чего надо оценить массу агрегата $\mathrm{M}(\mathrm{t})$.

В работах $[4,6,8]$ на основании экспериментальных исследований методами спектроскопии рассеяния фотонов получены зависимости, описывающие присоединение коллоидных частиц к ФА, а также разрушение ФА под действием скорости сдвига G. Учет разрушения и роста ФА позволяет дополнить уравнение (1) уравнением, позволяющим определять величину радиуса $\mathrm{R}$ в момент времени $\mathrm{t}$ :

$$
n d M / d t=K_{l}(R / a) N n-n K_{2} G a^{2}(R / a)^{p}-n K_{3} a^{2}(R / a)^{S},
$$

где $\mathrm{N}$ - концентрация одиночных и мелких коллоидных частиц с $\mathrm{R} / \mathrm{a}<10[4,9]$, не обладающих структурой физического фрактала;

$\mathrm{K}_{1}(\mathrm{R} / \mathrm{a}) \mathrm{N} \mathrm{n}$ - скорость приращения массы ФА[4, 6];

$\mathrm{K}_{1}-$ константа скорости реакции, учитывающая вероятность присоединения коллоидной частицы при столкновении с ФА;

$\mathrm{nK}_{2} \mathrm{Ga}^{2}(\mathrm{R} / \mathrm{a})^{\mathrm{p}}$ - скорость отрыва коллоидных частиц от ФА в результате действия скорости сдвига $\mathrm{G}$, вызывающего напряжение сдвига $\eta_{\infty} \mathrm{Ga}^{2}$.;

$\mathrm{K}_{2}$ - константа скорости отрыва;

$\mathrm{nK}_{3} \mathrm{a}^{2}(\mathrm{R} / \mathrm{a})^{\mathrm{d}}$ - скорость теплового разрушения ФА, в результате теплового отрыва коллоидных частиц от поверхности площадью $\sim \mathrm{a}^{2}(\mathrm{R} / \mathrm{a})^{\mathrm{d}}$;

$\mathrm{K}_{3}-$ константа скорости теплового разрушения ФА. 
Поскольку соотношение между концентрациями ФА -n и одиночных, и мелких частиц оценивается как $\mathrm{n} / \mathrm{N}=10^{-2}-10^{-4}<<1$ [10], в формуле (3) можно считать суммарную концентрацию $\mathrm{N}$ одиночных и малых агрегатов, не обладающих структурой физического фрактала и не подверженных разрушению под действием скорости сдвига, постоянной величиной $\mathrm{N}=$ const.

Вводя новую переменную $R=(\mathrm{R} / \mathrm{a})$, для $(\mathrm{R} / \mathrm{a})>>1$ после упрощений получаем уравнение вида (5):

$$
D R^{D-1} d R / d t=K_{1} R N-K_{2} G R^{p}-K_{3} R^{d} .
$$

Уравнения (1), (5) представляют полную систему уравнений, позволяющих рассчитывать как стационарные значения, когда $(\mathrm{dM} / \mathrm{dt})=0$, так и зависящие от времени значения вязкости коллоидного раствора нефти. Следует отметить, что константы реакций $K_{1}, K_{2} u K_{3}$ зависят от $\mathrm{D}, \mathrm{Gt}, R$, но константы остаются постоянными в достаточно больших диапазонах изменений этих параметров, как будет экспериментально показано ниже.

Уравнение (5) представляет уравнение с неразделяющимися переменными, в качестве которых выступают $R$ и t, общего решения (5) в аналитическом виде при произвольных значениях $\mathrm{p}$ и $\mathrm{d}$ не существует. Однако (5) допускает частные решения в аналитическом виде, которые соответствуют конкретным физическим ситуациям.

Рассмотрим некоторые частные решения уравнения (4).

1. Средняя масса ФА постоянна $-(d M / d t)=0$, скоростью термического распада при данной температуре можно пренебречь, т.е. в этом случае $K_{3} R^{d} \ll K_{1} R N u K_{3} R^{d}<<K_{2} G R^{p}$.

Решение уравнения (4) в этом случае имеет вид:

$$
K_{1} R N=K_{2} G R^{p} .
$$


Откуда получаем зависимость аналогичную (2):

$$
R=\left(K_{c} N / K_{G}\right)(1 / G)^{1 / p-1} .
$$

Как будет показано ниже, величина $\mathrm{p}>1$. В этом случае при измерении зависимости $\eta(G)$ в условиях, когда регистрация $\eta$ при каждом новом значении $\mathrm{G}$ через интервал времени, достаточный для установления стационарного значения вязкости, получаем:

$$
\operatorname{Ln}\left(\eta / \eta_{\infty}-1\right)=A_{1}-(\lambda / p-1) \operatorname{Ln} G .
$$

Экспериментально полученные зависимости типа (6) представляют ломаную линию, состоящую из прямых отрезков, соответствующих достаточно большим интервалам значений G. На рисунке 1 приведен пример зависимости (6), полученной для образца нефти.

Измерения производились с помощью вискозиметра «Physica MCR 301» фирмы «Anton Paar» (Австрия) с измерительной системой в геометрии конус-плита. Измерения производились при атмосферном давлении в диапазоне температур выпадения твердой фазы, диапазон определялся методом дифференциальной сканирующей калориметрии.

Зависимость типа, приведенной на рисунке 1 , отражает изменение фрактальной размерности $\mathrm{D}$ в ходе уменьшения радиуса $R$ при разрушении ФА под действием скорости сдвига G. Такой вид зависимости показывает, что рост ФА происходил в условиях, когда плотность менялась скачкообразно во времени в ходе увеличения массы ФА [7]. 


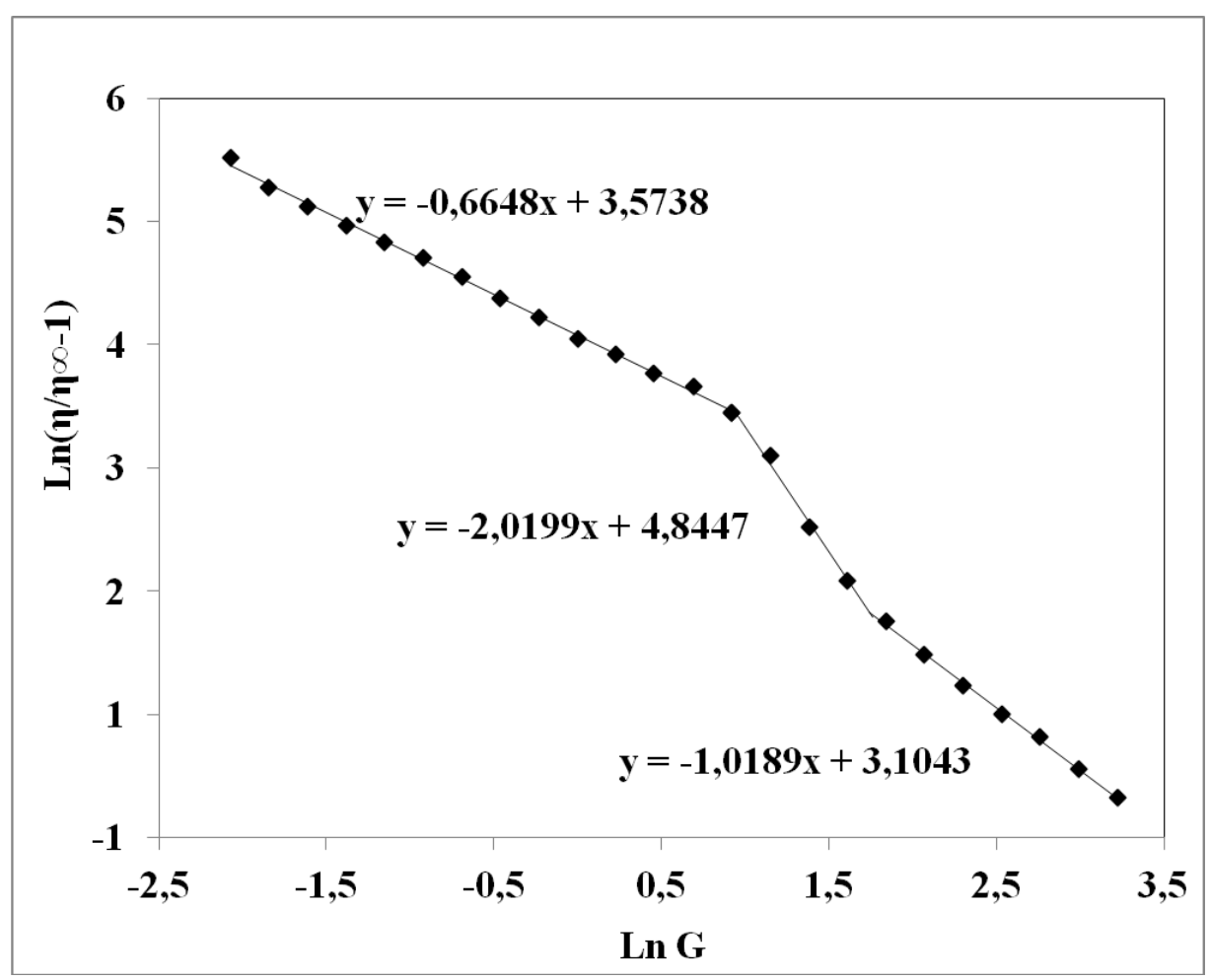

с ростом $\mathrm{G}$ величина ( $\lambda / \mathrm{P}-1)$ принимает значения: 0,$6648 ; 2,0199 ; 1,0189$;

точки - экспериментальные данные

Рисунок 1. Зависимость $\operatorname{Ln}\left(\eta / \eta_{\infty}-1\right), \operatorname{LnG}$, полученная для образца нефти при температуре $5{ }^{\circ} \mathrm{C}$

2. Коллоидная система в момент времени $t$ представляет одиночные и мелкие частицы, величинами $K_{2} G R^{p}$ u $K_{3} R^{d}$ можно пренебречь: $\left(K_{2} G R^{p}+K_{3} R^{d}\right)<<K_{1} R N$.

Для регистрации вязкости выбирается достаточно малая скорость сдвига $\mathrm{G}$, величину которой можно определить после предварительного получения зависимости $\eta(\mathrm{G})$ : величина $\mathrm{G}$ должна находиться в диапазоне значений $(\lambda / \mathrm{p}-1)>>0$. Перед началом измерений вязкости система была приведена в состояние полностью разрушенных ФА путем воздействия скоростью сдвига $1000 \mathrm{c}^{-1}$ в течение $4000 \mathrm{c}$, после чего произведены измерения вязкости во времени при скорости сдвига $1 \mathrm{c}^{-1}$. Эта скорость сдвига соответствовала области значений $(\lambda / \mathrm{p}-1)>0$, что было предварительно установлено экспериментально.

Уравнение (4) при условии, что $K_{2} G R^{p}+K_{3} R^{d} \quad<K_{l} R N$ имеет вид:

$$
(D / D-1) d R^{D-1}=K_{1} N d t
$$


Частное решение:

$$
\begin{gathered}
R=\left(C_{2} t\right)^{1 / D-1} \\
C_{2}=\left(K_{1} N(D-1 / D)\right) .
\end{gathered}
$$

Тогда

$$
\operatorname{Ln}\left(\eta / \eta_{\infty}-1\right)=A_{1}+(\lambda / D-1) \operatorname{Ln}(t) .
$$

На рисунке 2 приведена зависимость $\operatorname{Ln}\left(\eta / \eta_{\infty}-1\right), \operatorname{Ln}(\mathrm{t})$, полученная для образца нефти при температуре $10{ }^{\circ} \mathrm{C}$. Видно хорошее совпадение с линейной зависимостью, соответствующей формуле:

$$
\eta(t)=0,17\left(1+0,147 t^{0,33}\right) ; \quad \eta_{\infty}=0.17(\Pi a \cdot c),(\lambda / D-1)=0,33 .
$$

Колебания значений на начальном участке зависимости вызваны приборными особенностями, связанными с удержанием постоянного значения скорости сдвига в ходе измерений.

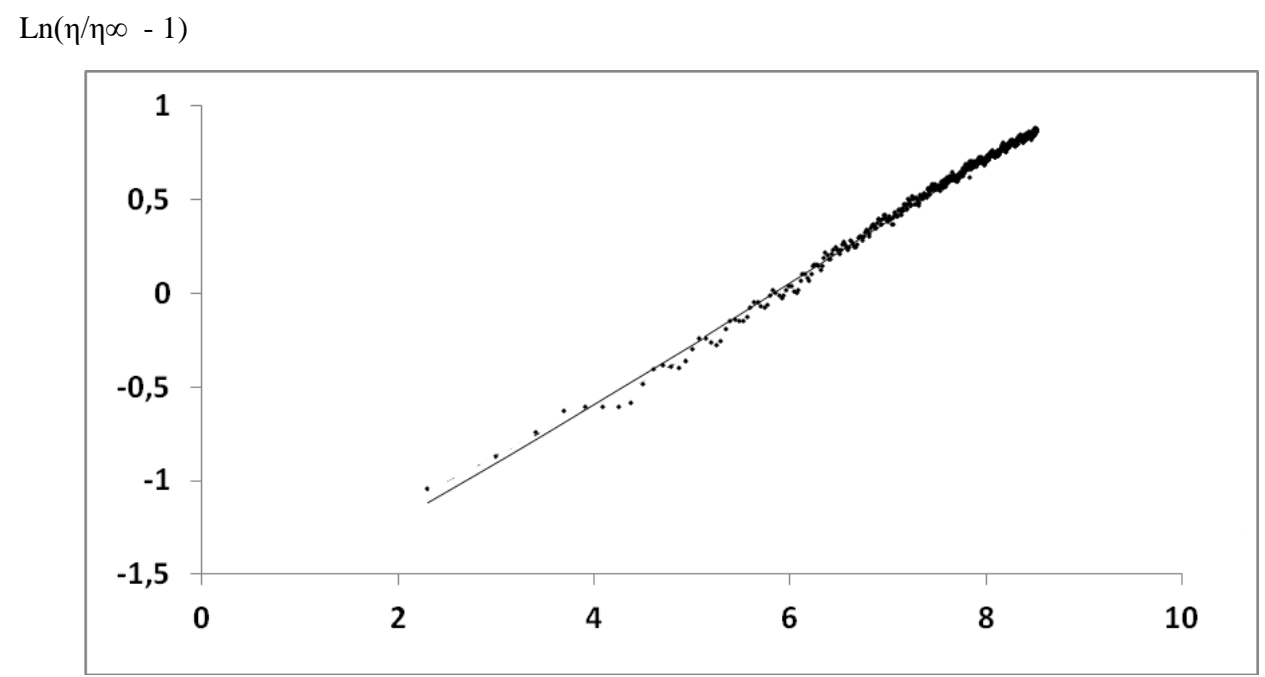

$\operatorname{Ln}(\mathrm{t})$

Релаксация вязкости при скачке скорости сдвига от $1000 \mathrm{c}^{-1}$ до $-1 \mathrm{c}^{-1}$.

Точки - экспериментальные данные

Рисунок 2. Зависимость $\operatorname{Ln}\left(\eta / \eta_{\infty}-1\right), \operatorname{Ln}(\mathrm{t})$ для образца нефти при температуре $10{ }^{\circ} \mathrm{C}$

3. Рассмотрим следующий предельный случай, когда первоначально покоящаяся в течение длительного времени нефть приводится в движение с некоторой скоростью сдвига. Это соответствует началу движения нефти 
в поровом пространстве при эксплуатации месторождения и/или процедурам извлечения нефти из емкостей хранения. В начальный момент времени мы имеем агрегаты больших размеров и на образец накладывается скорость сдвига G. Тогда при $K_{G}(\mathrm{G})^{\mathrm{s}} R^{\mathrm{p}}>K_{T} R^{\mathrm{d}}-K_{c} R \mathrm{~N}$ для (5) имеем:

$$
R^{D-1} d R / d t=-K_{2} G R^{p}
$$

При $\mathrm{p}>\mathrm{D}$

$$
(D / p-D) d R^{D-p}=K_{2} G d t
$$

Тогда частное решение имеет вид:

$$
\begin{gathered}
R=\left(C_{3} t\right)^{1 / \mathrm{D}-\mathrm{p}}, \\
C_{3}=\left(\left(D / P-D^{)} K_{2} G\right) .\right.
\end{gathered}
$$

Тогда

$$
\ln \left(\eta / \eta_{\infty}-1\right)=A_{2}+(1 / D-p) \operatorname{Ln}(t)
$$

На рисунке 3 приведен пример измерения зависимости вязкости от времени для случая, когда на образец нефти, длительное время находившемся в покое, в момент времени $\mathrm{t}=0$ наложена скорость сдвига $1000 \mathrm{c}^{-1}$.

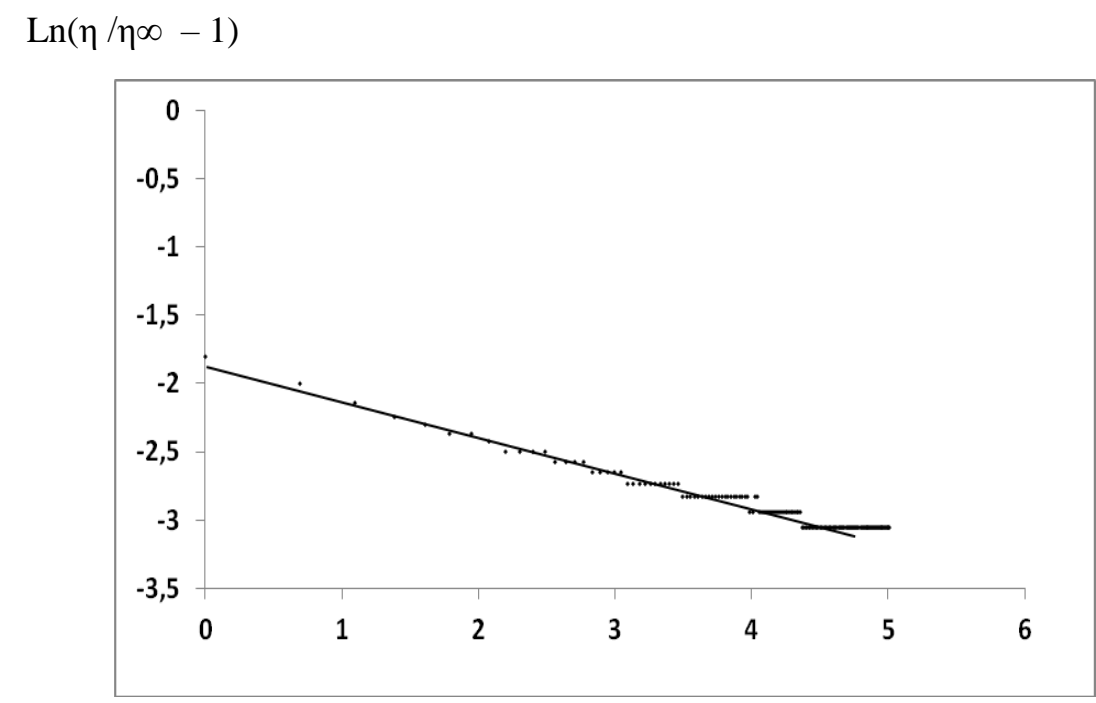

Наклон прямой $(1 / \mathrm{D}-\mathrm{P})=-0,24$;

температура образца $10{ }^{0} \mathrm{C}$;

Точки - экспериментальные данные

Рисунок 3. Релаксация вязкости при скачке скорости сдвига G $0 \rightarrow 1000 \mathrm{c}^{-1}$ 
Зависимость $\operatorname{Ln}\left(\eta / \eta_{\infty}-1\right)$ от $\operatorname{Ln}(\mathrm{t})$ для $\mathrm{t}>1 \mathrm{c}$ имеет линейный вид, соответствующий $\eta(\mathrm{t})=0,17\left(1+0,144 \mathrm{t}^{-0,24}\right) ;(1 / \mathrm{D}-\mathrm{P})=-0,24$, $\eta_{\infty}=0,17$ (Па•с), температура образца $10{ }^{\circ} \mathrm{C}$.

В нефти и нефтепродуктах, содержащих механические примеси, $\mathrm{n}$ является постоянной величиной, совпадающей с концентрацией центров роста, в роли которых преимущественное положение занимают частицы оксидов железа $[1,2,11,12]$. Это обусловлено высокой энергией адсорбции асфальтенов на поверхности оксидов, превышающей энергию взаимодействия асфальтенов, являющихся центрами формирования коллоидных частиц нефти. Магнитные частицы оксидов железа присутствуют в виде одиночных частиц размером порядка нескольких единиц и сотен нанометров, так и агрегатов, в составе которых могут находиться несколько десятков-сотен отдельных частиц $[12,13]$. При этом как ФА агрегаты, так и отдельные частицы оксидов являются центрами роста фрактальных агрегатов коллоидных частиц нефти. Обработка магнитным полем сопровождается дроблением агрегатов частиц оксидов железа - значительным увеличением n [13], что эквивалентно добавлению магнитных частиц железа, которое сопровождается снижением вязкости [14]. Магнитная обработка вызывает уменьшение размеров коллоидных образований в нефти $\mathrm{R}$ [15]. Из [16] следует, что после магнитной обработки произведение $\mathrm{nR} \approx$ const.

Как следует из формулы (1), вязкость пропорциональна $\mathrm{n}(\mathrm{R} / \mathrm{a})^{\lambda}$. Поскольку $3 \leq \lambda \leq 4$ [1], то рост величины $\mathrm{n}$ с учетом $\mathrm{nR} \approx$ const сопровождается уменьшением $\mathrm{n}(\mathrm{R} / \mathrm{a})^{\lambda}$ и, как следствие, уменьшением вязкости ๆ. В [13] наблюдалось увеличение $\mathrm{n}$ примерно в 3 раза, из чего следует, что радиус ФА должен снизиться в 3 раза после магнитной обработки. Полагая $\lambda=3$ получаем, что произведение $\mathrm{n}(\mathrm{R} / \mathrm{a})^{\lambda}$ после $\mathrm{MO}$ составляет 1/9 от исходного, что соответствует снижению вязкости. Величина снижения вязкости зависит от концентрации наноразмерных магнитных частиц оксидов железа и, например, для нефти месторождений 
Вьетнама снижение вязкости после магнитной обработки достигает $70-80 \%[17]$.

\section{Выводы}

Предложена система уравнений, описывающих зависимость вязкости тяжелой нефти от времени, скорости сдвига и концентрации центров формирования агрегатов коллоидных частиц - магнитных частиц оксидов железа.

Система уравнений содержит дифференциальное уравнение с неразделяющимися переменными, не имеющее общего аналитического решения. Справедливость дифференциального уравнения подтверждена экспериментально для трех типов частных решений, соответствующих различным физическим состояниям дисперсной составляющей нефти.

Предложенная теория обосновывает снижение вязкости после обработки магнитным полем нефти, содержащей примеси магнитных частиц железа.

Автор выражает благодарность Клепикову И.А. за помощь в проведении измерений вязкости.

Работа выполнена в рамках государственного задания (тема «Фундаментальный базис инноваџионных технологий нефтяной и газовой промыциленности», № AАAA-A16-116031750016-3).

\section{Список используемых источников}

1. Lesin V.I., Koksharov Yu. A., Khomutov G.B. Viscosity of Liquid Suspensions with Fractal Aggregates: Magnetic Nanoparticles in Petroleum Colloidal Structures // Colloids and Surfaces A: Physicochem. Eng. Aspects. 2011. Vol. 392. pp. 88-94. 
2. Лесин В.И., Лесин С.В. Фрактальная формула зависимости вязкости неньютоновской жидкости от градиента скорости // Нефтяное хозяйство. 2012. № 3. C. 46-48.

3. Лесин В.И., Клепиков И.А., Лесин С.В. Использование сдвигового воздействия для снижения вязкости нефти // Актуальные проблемы нефти и газа. 2016. № 1 (13).

4. Жюльен Р. Фрактальные агрегаты // Успехи физических наук. 1989. T. 157. № 2. С. 339 -357.

5. Лесин В.И. Особенности релаксации вязкости неньютоновской нефти после воздействия градиентами скорости и магнитными полями // Нефтепромысловое дело. 2008. № 1. С. 43-46.

6. Sontag R.C., Russel W.B. Structure and Breakup of Flocs Subjected to Fluid Stresses. 1 Shear Experiments // Journal of Colloid and Interface Science. 1986. Vol. 113. No. 2. pp. 399-413.

7. Ролдугин В.И. Свойства фрактальных систем // Успехи химии. 2003. T. 72. № 11, С. 1027-1054.

8. Barthelmes G., Prastinis S.E., Buggisch H. Particle Size Distribution and Viscosity of Suspensions Undergoing Shear-Induced Coagulation and Fragmentation // Chemical Engineering Science. 2003. Vol. 58. pp. 2893-2902. DOI:10.1016/s0009-2509(03)00133-7.

9. Puertas A.M., Fernandez-Barbero A., de las Nieves F.J. Internal Structure of Clasters from Charge Heterocoagulation // Journal of Colloid and Interface Science. 2004. Vol. 274. pp. 346-348.

10. Долгоносов Б.М. Параметры равновесного спектра частиц в коагулирующей системе с распадом агрегатов // Коллоидный журнал. 2001. T. 63. № 1. С. 39-42.

11. Nassar N.N., Betancur S., Acevedo S., et al. Development of a Population Balance Model to Describe the Influence of Shear and Nanoparticles on the Aggregation and Fragmentation of Asphaltene Aggregates // Industrial and Engineering Chemistry Research. 2015. Vol. 54. pp. 8201-8211. 
12. Pastor-Satorras R., Rubi J.M. Fractal Properties of Cluster of Colloidal Magnetic Particles // Progr. Colloid. Polym. Sci., 1998. Vol. 110. pp. 29-33.

13. Лесин В.И. Физико-химическая модель изменения нефтевытесняющих свойств воды после ее магнитной обработки // Нефтепромысловое дело. 2001. № 3. С. 15-17.

14. Aristizabal-Fontal J.E., Cortes F.B., Franco C.A. Viscosity Reduction of Extra Heavy Crude Oil by Magnetite Nanoparticle-Based Ferrofluids // Adsorption Science \& Technology (Special Collection: III Workshop on Adsorption, Catalysis and Porous Materials). 2017. pp. 1-23. DOI: 10.1177/0263617417704309.

15. Лоскутова Ю.В., Юдина Н.В. Влияние постоянного магнитного поля на реологические свойства высокопарафинистых нефтей // Коллоидный журнал. 2003. Т. 65. № 4. С. 510-515.

16. Мартынова О.И., Гусев Я.Т., Леонтьев Е.А. К вопросу о механизме влияния магнитного поля на водные растворы солей // Успехи физических наук. 1969. Т. 98. Вып. 1. С. 195-199.

17. Tung N.P., Vinh N.Q., Phong N.T.P., Long B.Q.K., Hung P.V. Perspective for Using Nd-Fe-B Magnets as a Tool for the Improvement of the Production and Transportation of Vietnamese Crude Oil with High Paraffin Content // Physica B. 2003. Vol. 327. pp. 443-447.

\section{References}

1. Lesin V.I., Koksharov Yu.A., Khomutov G.B. Viscosity of Liquid Suspensions with Fractal Aggregates: Magnetic nanoparticles in Petroleum Colloidal Structures. Colloids and Surfaces A: Physicochem. Eng. Aspects, 2011, Vol. 392, pp. 88-94.

2. Lesin V.I., Lesin S.V. Fraktal'naya formula zavisimosti vyazkosti nen'yutonovskoi zhidkosti ot gradienta skorosti [Fractal Formula for The Dependence of The Viscosity of a Non-Newtonian Fluid on The Velocity Gradient]. Neftyanoe khozyaistvo - Oil Industry, 2012, No. 3, pp. 46-48. [in Russian]. 
3. Lesin V.I., Klepikov I.A., Lesin S.V. Ispol'zovanie sdvigovogo vozdeistviya dlya snizheniya vyazkosti nefti [Using a Shear Rate Effect for Oil Viscosity Reduction]. Aktual'nye problemy nefti i gaza - Actual Problems Of Oil and Gas, 2016, No. 1 (13). [in Russian].

4. Zhyul'en R. Fraktal'nye agregaty [Fractal Aggregates]. Uspekhi fizicheskikh nauk - Actual Problems of Oil and Gas, 1989, Vol. 157, No. 2, pp. 339 -357. [in Russian].

5. Lesin V.I. Osobennosti relaksatsii vyazkosti nen'yutonovskoi nefti posle vozdeistviya gradientami skorosti i magnitnymi polyami [Features of Relaxation of Viscosity of Non-Newtonian Oil after Exposure to Velocity Gradients and Magnetic Fields]. Neftepromyslovoe delo - Oilfield Engineering, 2008, No. 1, pp. 43-46. [in Russian].

6. Sontag R.C., Russel W.B. Structure and Breakup of Flocs Subjected to Fluid Stresses. 1 Shear Experiments. Journal of Colloid and Interface Science, 1986, Vol. 113, No. 2, pp. 399-413.

7. Roldugin V.I. Svoistva fraktal'nykh sistem [Properties of Fractal Systems]. Uspekhi fizicheskikh nauk - Actual Problems of Oil and Gas, 2003, Vol. 72, No. 11, pp. 1027-1054. [in Russian].

8. Barthelmes G., Prastinis S.E., Buggisch H. Particle Size Distribution and Viscosity of Suspensions Undergoing Shear-Induced Coagulation and Fragmentation. Chemical Engineering Science, 2003, Vol. 58, pp. 2893-2902. DOI:10.1016/s0009-2509(03)00133-7.

9. Puertas A.M., Fernandez-Barbero A., de las Nieves F.J. Internal Structure of Clasters from Charge Heterocoagulation. Journal of Colloid and Interface Science, 2004, Vol. 274, pp. 346-348.

10. Dolgonosov B.M. Parametry ravnovesnogo spektra chastits v koaguliruyushchei sisteme s raspadom agregatov [Parameters of the Equilibrium Spectrum of Particles in the Coagulating System with the Decay of Aggregates]. Kolloidnyi zhurnal - Colloid Journal, 2001, Vol. 63, No. 1, pp. 39-42. [in Russian]. 
11. Nassar N.N., Betancur S., Acevedo S., et al. Development of a Population Balance Model to Describe the Influence of Shear and Nanoparticles on the Aggregation and Fragmentation of Asphaltene Aggregates. Industrial and Engineering Chemistry Research, 2015, Vol. 54, pp. 8201-8211.

12. Pastor-Satorras R., Rubi J.M. Fractal Properties of Cluster of Colloidal Magnetic Particles. Progr. Colloid. Polym. Sci., 1998, Vol. 110, pp. 29-33.

13. Lesin V.I. Fiziko-khimicheskaya model' izmeneniya neftevytesnyayushchikh svoistv vody posle ee magnitnoi obrabotki [PhysicoChemical Model of Changes in Oil-Displacing Properties of Water After its Magnetic Treatment]. Neftepromyslovoe delo - Oilfield Engineering, 2001, No. 3, pp. 15-17. [in Russian].

14. Aristizabal-Fontal J.E., Cortes F.B., Franco C.A. Viscosity Reduction of Extra Heavy Crude Oil by Magnetite Nanoparticle-Based Ferrofluids. Adsorption Science \& Technology (Special Collection: III Workshop on Adsorption, Catalysis and Porous Materials), 2017, pp. 1-23. DOI: $10.1177 / 0263617417704309$

15. Loskutova Yu.V., Yudina N.V. Vliyanie postoyannogo magnitnogo polya na reologicheskie svoistva vysokoparafinistykh neftei [Influence of a Constant Magnetic Field on Rheological Properties of High-Paraffin Oil]. Kolloidnyi zhurnal - Colloid Journal, 2003, Vol. 65, No. 4, pp. 510-515. [in Russian].

16. Martynova O.I., Gusev Ya.T., Leont'ev E.A. K voprosu o mekhanizme vliyaniya magnitnogo polya na vodnye rastvory solei [On the Mechanism of Influence of the Magnetic Field on Aqueous Solutions of Salts]. Uspekhi fizicheskikh nauk - Actual Problems of Oil and Gas, 1969, Vol. 98, Issue 1, pp. 195-199. [in Russian].

17. Tung N.P., Vinh N.Q., Phong N.T.P., Long B.Q.K., Hung P.V. Perspective for Using $\mathrm{Nd}-\mathrm{Fe}-\mathrm{B}$ Magnets as a Tool for the Improvement of the Production and Transportation of Vietnamese Crude Oil with High Paraffin Content. Physica B, 2003, Vol. 327, pp. 443-447. 


\section{Сведения об авторе}

\section{About the author}

Лесин Виктор Иванович, канд. физ.-мат. наук, ведущий научный сотрудник, Институт проблем нефти и газа Российской Академии наук, г. Москва, Российская Федерация

Viktor I. Lesin, Candidate of Physics and Mathematics Sciences, Leading Researcher, Institute of Oil and Gas Problems, Russian Academy of Sciences, Moscow, Russian Federation

e-mail: vilesin@inbox.ru 